Article

\title{
Projective Exponential Synchronization for a Class of Complex PDDE Networks with Multiple Time Delays
}

\section{Chengdong Yang ${ }^{1,4, *}$, Jianlong Qiu ${ }^{2, *}$, Tongxing Li ${ }^{1}$, Ancai Zhang ${ }^{3}$ and Xiangyong Chen ${ }^{2}$}

1 School of Informatics, Linyi University, Linyi 276005, China; E-Mail: litongx2007@163.com

2 School of Science, Linyi University, Linyi 276005, China; E-Mail: cxy8305@163.com

3 School of Automobile Engineering, Linyi University, Linyi 276005, China;

E-Mail: zhangancai@lyu.edu.cn

4 School of Automation, Southeast University, Nanjing 210096, China

* Authors to whom correspondence should be addressed; E-Mails: yangchengdong@lyu.edu.cn (C.Y.); qiujianlong@lyu.edu.cn (J.Q.); Tel./Fax: +86-539-8766320 (C.Y).

Academic Editor: J. A. Tenreiro Machado

Received: 20 April 2015 / Accepted: 19 October 2015 / Published: 28 October 2015

\begin{abstract}
This paper addresses the problem of projective exponential synchronization for a class of complex spatiotemporal networks with multiple time delays satisfying the homogeneous Neumann boundary conditions, where the network is modeled by coupled partial differential-difference equations (PDDEs). A distributed proportional-spatial derivative (P-sD) controller is designed by employing Lyapunov's direct method and Kronecker product. The controller ensures the projective exponential synchronization of the PDDE network. The main result of this paper is presented in terms of standard linear matrix inequality (LMI). A numerical example is provided to show the effectiveness of the proposed design method.
\end{abstract}

Keywords: spatiotemporal behavior; partial differential-difference equation; projective exponential synchronization; linear matrix inequality 


\section{Introduction}

Complex networks have extensive promising applications in many areas, including signal processing, moving image processing, speed detection of moving subjects, logistics networks, EPC system networks, associative memories, etc. This has inspired many researchers to study the problems presented by complex networks (see [1-4] and the references cited therein).

Among them, various synchronizations of complex networks have especially attracted researchers' attention in the past few decades [5-7]. For example, cluster synchronization, pinning synchronization, projective synchronization, impulsive synchronization, adaptive synchronization, and many other outstanding research works have been presented (e.g., [8-11]). Among all kinds of synchronization schemes, projective synchronization is the best known one because of its wide use in signal processing [12-14].

Time delays are inevitable in the real applications of complex networks. Time delays may cause bifurcation, oscillation, divergence or instability of complex networks [15-17]. Thus, it is extremely important to study the dynamics of complex networks considering time delays, which has become a hotly discussed issue in the area of complex networks studies [18-23]. In addition, a kind of complex networks called complex spatiotemporal networks (e.g., biological systems [24]) have also attracted researchers' attention in the past few years. The outputs, inputs, and process states and relevant parameters of this kind of complex networks may vary temporally and spatially. Complex spatiotemporal networks can usually be modeled by coupled partial differential equations (PDEs). Recently, many outstanding research works have been carried out on the synchronization of complex spatiotemporal networks recently [22-35].

It is worth mentioning that the model of complex networks with time delays is expressed by coupled partial differential-difference equations (PDDEs). Compared to the complex PDE networks themselves, the dynamics of complex PDDE networks are more difficult to analyze. Up to now, few results are available on the projective exponential synchronization of complex PDDE networks with multiple time delays. This is a very challenging problem, which motivates our study presented in this paper.

The objective of this paper is thus to address the problem of projective exponential synchronization for a class of complex PDDE networks with multiple time delays satisfying the homogeneous Neumann boundary conditions. In order to achieve the projective exponential synchronization of complex PDDE networks, a distributed proportional-spatial derivative (P-sD) controller is designed by using the Lyapunov direct method. The solution of the projective exponential synchronization problem then reduces to the feasibility of a linear matrix inequality (LMI). Moreover, a numerical simulation is provided to illustrate the effectiveness of the proposed synchronization criteria.

The remainder of this paper is organized as follows: the problem formulation and preliminaries are given in the next section. Section 3 studies a distributed P-sD state feedback controller design for projective exponential synchronization of a complex PDDE network with multiple time delays in terms of LMI. Section 4 presents an example to illustrate the effectiveness of the proposed method and Section 5 offers the conclusions. 


\subsection{Notations}

The following notations will be used in what follows. $\mathfrak{R}, \mathfrak{R}^{n}$ and $\mathfrak{R}^{m \times n}$ denote the set of all real numbers, $n$-dimensional Euclidean space and the set of all $m \times n$ matrices, respectively. $\mathcal{N}$ is the set of nodes. $\otimes$ is the Kronecker product for matrices. The identity matrix with appropriate dimensions is denoted by $\boldsymbol{I}$. For a symmetric matrix $\boldsymbol{M}, \boldsymbol{M}>0$ and $\boldsymbol{M}<0$ respectively stands for $\boldsymbol{M}$ is positive definite and negative definite.

The superscript " $T$ " is used for the transpose of a vector or a matrix. The symbol "*" is used as an ellipsis in matrix expressions that are induced by symmetry, e.g.,:

$$
\left[\begin{array}{cc}
\boldsymbol{R}+[\boldsymbol{M}+\boldsymbol{N}+*] & \boldsymbol{X} \\
* & \boldsymbol{Y}
\end{array}\right] \triangleq\left[\begin{array}{cc}
\boldsymbol{R}+\left[\boldsymbol{M}+\boldsymbol{N}+\boldsymbol{M}^{T}+\boldsymbol{N}^{T}\right] & \boldsymbol{X} \\
\boldsymbol{X}^{T} & \boldsymbol{Y}
\end{array}\right] .
$$

\section{Preliminaries and Problem Formulation}

Consider a class of complex PDDE networks with multiple time delays of the following form:

$$
\left\{\begin{array}{rl}
\boldsymbol{y}_{i, t}(x, t)= & \boldsymbol{\Theta}_{1} \boldsymbol{y}_{i, x x}(x, t)+\Theta_{1 d} \boldsymbol{y}_{i, x x}\left(x, t-\tau_{1}\right)+\boldsymbol{\Theta}_{2} \boldsymbol{y}_{i, x}(x, t)+\boldsymbol{\Theta}_{2 d} \boldsymbol{y}_{i, x}\left(x, t-\tau_{2}\right)+\boldsymbol{A} \boldsymbol{y}_{i}(x, t) \\
& +\boldsymbol{A}_{d} \boldsymbol{y}_{i}\left(x, t-\tau_{3}\right)+c \sum_{j=1}^{N} g_{i j} \boldsymbol{y}_{j}(x, t)+\boldsymbol{B} \boldsymbol{u}_{i}(x, t),(x, t) \in[0, L] \times[0,+\infty) \\
\left.\boldsymbol{y}_{i, x}(x, t)\right|_{x=0}=\left.\boldsymbol{y}_{i, x}(x, t)\right|_{x=L}=0, t \in[-\tau,+\infty) & \boldsymbol{y}_{i}(x, t)=\phi_{i}(x, t),(x, t) \in[0, L] \times[-\tau, 0]
\end{array},\right.
$$

where $\mathcal{N} \triangleq\{1,2, \cdots, N\}$ is the set of nodes. $\boldsymbol{y}_{i}(x, t) \in \mathfrak{R}^{n}$ and $\boldsymbol{u}_{i}(x, t) \in \mathfrak{R}^{m}, i \in \mathcal{N}$, are the state and distributed control input of the $i$-th node, respectively. $\Theta_{1} \in \mathfrak{R}^{n \times n}, \Theta_{2} \in \mathfrak{R}^{n \times n}, \boldsymbol{A} \in \mathfrak{R}^{n \times n}, \boldsymbol{B} \in \mathfrak{R}^{n \times m}$ are known matrices. $\tau_{1}>0, \tau_{2}>0, \tau_{3}>0$ are time delays in the network and $\tau \triangleq \max \left\{\tau_{1}, \tau_{2}, \tau_{3}\right\}$ denotes the largest time delay throughout the paper. $x \in[0, L] \subset \Re$ and $t \in[0, \infty)$ are the spatial position and time, respectively. $\phi_{t}(x, t), t \in[-\tau, 0], i \in \mathcal{N}$, is the initial value of the $i$-th node. $c$ is a known scalar describing the coupling strength. $\boldsymbol{G}=\left(g_{i j}\right)_{N \times N}, i, j \in \mathcal{N}$, is the coupling configuration matrix representing the topological structure of the network defined to be diffusive, where $g_{i j}>0(i \neq j)$, $g_{i i}=-\sum_{j=1, j \neq i}^{N} g_{i j}$. The coupling matrix $\boldsymbol{G}$ is not required to be symmetric or irreducible.

Assume that there is an isolated node $s(x, t)$ satisfying:

$$
\left\{\begin{array}{l}
\quad \begin{array}{l}
\boldsymbol{s}_{t}(x, t)=\Theta_{1} \boldsymbol{s}_{x x}(x, t)+\Theta_{1 d} \boldsymbol{s}_{x x}\left(x, t-\tau_{1}\right)+\Theta_{2} \boldsymbol{s}_{x}(x, t)+\Theta_{2 d} \boldsymbol{s}_{x}\left(x, t-\tau_{2}\right)+\boldsymbol{A s}(x, t) \\
\quad+\boldsymbol{A}_{d} \boldsymbol{s}(x, t-\tau),(x, t) \in[0, L] \times[0,+\infty) \\
\left.\boldsymbol{s}_{x}(x, t)\right|_{x=0}=\boldsymbol{s}_{x}(x, t)_{x=L}=0, t \in[-\tau,+\infty) \\
\boldsymbol{s}(x, t)=\boldsymbol{s}_{0}(x, t),(x, t) \in[0, L] \times[-\tau, 0]
\end{array},
\end{array}\right.
$$

This paper aims to design a controller to make the complex PDDE network of Equation (1) proportionally synchronize onto the desired state of Equation (2). In order to investigate the projective synchronization of the complex PDDE network, the stability of the zero solution of the error system needs to be studied. Define the synchronization error of the $i$-th node to be:

$$
\boldsymbol{e}_{i}(x, t) \triangleq \boldsymbol{y}_{i}(x, t)+\alpha \mathbf{s}(x, t),
$$


where $\alpha$ is a desired scaling factor. Thus, it is not difficult to get the synchronization error system of the complex PDDE network as:

$$
\left\{\begin{aligned}
\quad \begin{array}{rl}
\boldsymbol{e}_{i, t}(x, t)= & \boldsymbol{\Theta}_{1} \boldsymbol{e}_{i, x x}(x, t)+\boldsymbol{\Theta}_{1 d} \boldsymbol{e}_{i, x x}\left(x, t-\tau_{1}\right)+\boldsymbol{\Theta}_{2} \boldsymbol{e}_{i, x}(x, t)+\boldsymbol{\Theta}_{2 d} \boldsymbol{e}_{i, x}\left(x, t-\tau_{2}\right)+\boldsymbol{A} \boldsymbol{e}_{i}(x, t) \\
& +\boldsymbol{A}_{d} \boldsymbol{e}_{i}\left(x, t-\tau_{3}\right)+c \sum_{j=1}^{N} g_{i j} \boldsymbol{e}_{j}(x, t)+\boldsymbol{B u}_{i}(x, t),(x, t) \in[0, L] \times[0,+\infty) \\
& \left.\boldsymbol{e}_{i, x}(x, t)\right|_{x=0}=\left.\boldsymbol{e}_{i, x}(x, t)\right|_{x=L}=0, t \in[-\tau,+\infty) \\
\boldsymbol{e}_{i}(x, t)=\boldsymbol{e}_{i, 0}(x, t),(x, t) \in[0, L] \times[-\tau, 0]
\end{array},
\end{aligned}\right.
$$

where $\boldsymbol{e}_{i, 0}(x) \triangleq \boldsymbol{y}_{i, 0}(x)+\alpha \boldsymbol{s}_{0}(x)$.

To achieve the synchronization of the complex PDDE network of Equation (1), we consider a distributed P-sD feedback controller:

$$
\boldsymbol{u}_{i}(x, t)=\boldsymbol{K}_{1} \boldsymbol{e}_{i}(x, t)+\boldsymbol{K}_{2} \boldsymbol{e}_{i, x}(x, t), i \in \mathcal{N},
$$

where $\boldsymbol{K}_{1}$ and $\boldsymbol{K}_{2}$ are real $m \times n$ matrices to be determined. It has been pointed out in [32,34-37] that the controller of Equation (9) can provide more convenient spatial performance. The signal $\boldsymbol{e}_{i, x}(x, t)$ in the controller of Equation (9) can be obtained via the finite difference method. Substituting Equation (9) into Equation (8) yields the closed-loop synchronization error system of the complex PDDE network:

$$
\left\{\begin{array}{rl}
\quad \begin{array}{rl}
\boldsymbol{e}_{i, t}(x, t)= & \boldsymbol{\Theta}_{1} \boldsymbol{e}_{i, x x}(x, t)+\boldsymbol{\Theta}_{1 d} \boldsymbol{e}_{i, x x}\left(x, t-\tau_{1}\right)+\boldsymbol{\Theta}_{2} \boldsymbol{e}_{i, x}(x, t)+\boldsymbol{\Theta}_{2 d} \boldsymbol{e}_{i, x}\left(x, t-\tau_{2}\right)+\left(\boldsymbol{A}+\boldsymbol{B} \boldsymbol{K}_{1}\right) \boldsymbol{e}_{i}(x, t) \\
& +\boldsymbol{A}_{d} \boldsymbol{e}_{i}\left(x, t-\tau_{3}\right)+c \sum_{j=1}^{N} g_{i j} \boldsymbol{e}_{j}(x, t)+\boldsymbol{B K}_{2} \boldsymbol{e}_{i, x}(x, t),(x, t) \in[0, L] \times[0,+\infty)
\end{array} \\
\left.\boldsymbol{e}_{i, x}(x, t)\right|_{x=0}=\left.\boldsymbol{e}_{i, x}(x, t)\right|_{x=L}=0, t \in[-\tau,+\infty) \\
\boldsymbol{e}_{i}(x, t)=\boldsymbol{e}_{i, 0}(x, t),(x, t) \in[0, L] \times[-\tau, 0]
\end{array} .\right.
$$

Hence, the aim of this paper is to design $\boldsymbol{K}_{1}$ and $\boldsymbol{K}_{2}$ for Equation (6) such that the complex PDDE network of Equation (1) exponentially synchronizes the trajectory of Equation (2). To this end, we firstly introduce the following definition of projective exponential synchronization. The definition is useful for the sequel analysis and design.

Definition 1. Given a constant $\rho>0$, the complex PDDE systems of Equation (1) are said to be projective exponentially synchronized, if there exists a constant $\sigma>0$ such that the following inequality holds:

$$
\|\boldsymbol{e}(\cdot, t)\|_{2}^{2} \leq \sigma \exp (-2 \rho t)\left\|\boldsymbol{e}_{0}(\cdot)\right\|_{2}^{2}, \forall t \geq 0
$$

By transforming the complex PDDE systems of Equation (1) with state vector to the synchronization errors of Equation (3), the synchronization of Equation (1) turns into the projective exponential stabilization of Equation (3). 


\section{Distributed P-sD State Feedback Control Design for Projective Exponential Synchronization}

By using Lyapunov's direct method, this section will present an LMI-based sufficient condition on the projective exponential synchronization of the complex PDDE network of Equation (1). First, we consider a Lyapunov functional for the closed-loop synchronization error system of Equation (6):

$$
\begin{aligned}
V(t) & =\sum_{i=1}^{N} \int_{0}^{L} \boldsymbol{e}_{i}^{T}(x, t) \boldsymbol{P} \boldsymbol{e}_{i}(x, t) d x+\sum_{i=1}^{N} \int_{0}^{L} \int_{t-\tau_{1}}^{t} \boldsymbol{e}_{i, x}^{T}(x, \rho) \boldsymbol{R}_{1} \boldsymbol{e}_{i, x}(x, \rho) d \rho d x \\
& +\sum_{i=1}^{N} \int_{0}^{L} \int_{t-\tau_{2}}^{t} \boldsymbol{e}_{i, x}^{T}(x, \rho) \boldsymbol{R}_{2} \boldsymbol{e}_{i, x}(x, \rho) d \rho d x+\sum_{i=1}^{N} \int_{0}^{L} \int_{t-\tau_{3}}^{t} \boldsymbol{e}_{i}^{T}(x, \rho) \boldsymbol{R}_{3} \boldsymbol{e}_{i}(x, \rho) d \rho d x
\end{aligned},
$$

where $\boldsymbol{P}>0, \boldsymbol{R}_{k}>0, k \in\{1,2,3\}$ are real symmetric $n \times n$ matrices to be determined. Based on the property of the Kronecker product for matrices and integrating by parts, a simple calculation gives the time derivative of $V(t)$ that:

$$
\begin{aligned}
\dot{V}(t)= & -\int_{0}^{L} \boldsymbol{e}_{x}^{T}(x, t)\left[\boldsymbol{I}_{N} \otimes \boldsymbol{P} \boldsymbol{\Theta}_{1}+*\right] \boldsymbol{e}_{x}(x, t) d x-\int_{0}^{L} \boldsymbol{e}_{x}^{T}(x, t)\left[\boldsymbol{I}_{N} \otimes \boldsymbol{P} \boldsymbol{\Theta}_{1 d}+*\right] \boldsymbol{e}_{x}\left(x, t-\tau_{1}\right) d x \\
& +2 \int_{0}^{L} \boldsymbol{e}^{T}(x, t)\left(\boldsymbol{I}_{N} \otimes \boldsymbol{P} \boldsymbol{\Theta}_{2}\right) \boldsymbol{e}_{x}(x, t) d x+2 \int_{0}^{L} \boldsymbol{e}^{T}(x, t)\left(\boldsymbol{I}_{N} \otimes \boldsymbol{P} \boldsymbol{\Theta}_{2 d}\right) \boldsymbol{e}_{x}\left(x, t-\tau_{2}\right) d x \\
& +\int_{0}^{L} \boldsymbol{e}^{T}(x, t)\left[\boldsymbol{I}_{N} \otimes\left(\boldsymbol{P} \boldsymbol{A}+\boldsymbol{P B} \boldsymbol{K}_{1}\right)+*\right] \boldsymbol{e}(x, t) d x+2 \int_{0}^{L} \boldsymbol{e}^{T}(x, t)\left(\boldsymbol{I}_{N} \otimes \boldsymbol{P} \boldsymbol{A}_{d}\right) \boldsymbol{e}\left(x, t-\tau_{3}\right) d x \\
& +\int_{0}^{L} \boldsymbol{e}^{T}(x, t)[\boldsymbol{G} \otimes \boldsymbol{P}+*] \boldsymbol{e}(x, t) d x+\int_{0}^{L} \boldsymbol{e}_{x}^{T}(x, t)\left(\boldsymbol{I}_{N} \otimes \boldsymbol{R}_{1}\right) \boldsymbol{e}_{x}(x, t) d x \\
& -\int_{0}^{L} \boldsymbol{e}_{x}^{T}\left(x, t-\tau_{1}\right)\left(\boldsymbol{I}_{N} \otimes \boldsymbol{R}_{1}\right) \boldsymbol{e}_{x}\left(x, t-\tau_{1}\right) d x+\int_{0}^{L} \boldsymbol{e}_{x}^{T}(x, t)\left(\boldsymbol{I}_{N} \otimes \boldsymbol{R}_{2}\right) \boldsymbol{e}_{x}(x, t) d x \\
& -\int_{0}^{L} \boldsymbol{e}_{x}^{T}\left(x, t-\tau_{2}\right)\left(\boldsymbol{I}_{N} \otimes \boldsymbol{R}_{2}\right) \boldsymbol{e}_{x}\left(x, t-\tau_{2}\right) d x+\int_{0}^{L} \boldsymbol{e}^{T}(x, t)\left(\boldsymbol{I}_{N} \otimes \boldsymbol{R}_{3}\right) \boldsymbol{e}(x, t) d x \\
& -\int_{0}^{L} \boldsymbol{e}^{T}\left(x, t-\tau_{3}\right)\left(\boldsymbol{I}_{N} \otimes \boldsymbol{R}_{3}\right) \boldsymbol{e}\left(x, t-\tau_{3}\right) d x \\
= & \int_{0}^{L} \tilde{\boldsymbol{e}}^{T}(x, t) \Psi \tilde{\boldsymbol{e}}(x, t) d x
\end{aligned}
$$

where:

$$
\begin{aligned}
& \tilde{\boldsymbol{e}}(x, t) \triangleq\left[\boldsymbol{e}^{T}(x, t) \boldsymbol{e}_{x}^{T}(x, t) \boldsymbol{e}_{x}^{T}\left(x, t-\tau_{1}\right) \boldsymbol{e}_{x}^{T}\left(x, t-\tau_{2}\right) \boldsymbol{e}^{T}\left(x, t-\tau_{3}\right)\right]^{T}, \\
& \boldsymbol{e}(x, t) \triangleq\left[\begin{array}{llll}
\boldsymbol{e}_{1}^{T}(x, t) & \boldsymbol{e}_{2}^{T}(x, t) & \cdots & \boldsymbol{e}_{N}^{T}(x, t)
\end{array}\right]^{T} \in \mathfrak{R}^{n N}, \\
& \Psi \triangleq\left[\begin{array}{ccccc}
\Psi_{11} & \boldsymbol{I}_{N} \otimes\left(\boldsymbol{P} \Theta_{2}+\boldsymbol{P B} \boldsymbol{K}_{2}\right) & 0 & \boldsymbol{I}_{N} \otimes \boldsymbol{P} \Theta_{2 d} & \boldsymbol{I}_{N} \otimes \boldsymbol{P} \boldsymbol{A}_{d} \\
* & \boldsymbol{\Psi}_{22} & \boldsymbol{I}_{N} \otimes \boldsymbol{P} \Theta_{1 d} & 0 & 0 \\
* & * & -\boldsymbol{I}_{N} \otimes \boldsymbol{R}_{1} & 0 & 0 \\
* & * & * & -\boldsymbol{I}_{N} \otimes \boldsymbol{R}_{2} & 0 \\
* & * & * & * & -\boldsymbol{I}_{N} \otimes \boldsymbol{R}_{3}
\end{array}\right],
\end{aligned}
$$

in which:

$$
\begin{aligned}
& \Psi_{11} \triangleq \boldsymbol{I}_{N} \otimes\left[\boldsymbol{P} \boldsymbol{A}+\boldsymbol{P B} \boldsymbol{K}_{1}+*\right]+\boldsymbol{I}_{N} \otimes \boldsymbol{R}_{3}+c[\boldsymbol{G} \otimes \boldsymbol{P}+*], \\
& \Psi_{22} \triangleq-\boldsymbol{I}_{N} \otimes\left[\boldsymbol{P} \boldsymbol{\Theta}_{1}+*\right]+\boldsymbol{I}_{N} \otimes \boldsymbol{R}_{1}+\boldsymbol{I}_{N} \otimes \boldsymbol{R}_{2} .
\end{aligned}
$$

From the above analysis, we have the following theorem. 
Theorem 1. Consider the complex PDDE network of Equation (1). For a given scalar $\rho>0$, there exists a distributed controller with the form of Equation (5) such that the network of Equation (1) achieves projective exponential synchronization, if there exist $n \times n$ symmetric matrices $\boldsymbol{Q}>0$, $\boldsymbol{S}_{1}>0, \quad \boldsymbol{S}_{2}>0, \quad \boldsymbol{S}_{3}>0$, and $m \times n$ matrices $\boldsymbol{Y}_{1}, \quad \boldsymbol{Y}_{2}$ satisfying the following LMI:

$$
\Xi \triangleq\left[\begin{array}{ccccc}
\boldsymbol{\Xi}_{11} & \boldsymbol{I}_{N} \otimes\left(\boldsymbol{\Theta}_{2} \boldsymbol{Q}+\boldsymbol{B} \boldsymbol{Y}_{2}\right) & 0 & \boldsymbol{I}_{N} \otimes \boldsymbol{\Theta}_{2 d} \boldsymbol{Q} & \boldsymbol{I}_{N} \otimes \boldsymbol{A}_{d} \boldsymbol{Q} \\
* & \boldsymbol{\Xi}_{22} & \boldsymbol{I}_{N} \otimes \boldsymbol{\Theta}_{1 d} \boldsymbol{Q} & 0 & 0 \\
* & * & -\boldsymbol{I}_{N} \otimes \boldsymbol{S}_{1} & 0 & 0 \\
* & * & * & -\boldsymbol{I}_{N} \otimes \boldsymbol{S}_{2} & 0 \\
* & * & * & * & -\boldsymbol{I}_{N} \otimes \boldsymbol{S}_{3}
\end{array}\right]>0
$$

where:

$$
\begin{gathered}
\boldsymbol{\Xi}_{11} \triangleq \boldsymbol{I}_{N} \otimes\left[\boldsymbol{A} \boldsymbol{Q}+\boldsymbol{B} \boldsymbol{Y}_{1}+*\right]+\boldsymbol{I}_{N} \otimes \boldsymbol{S}_{3}+c[\boldsymbol{G} \otimes \boldsymbol{Q}+*], \\
\boldsymbol{\Xi}_{22} \triangleq-\boldsymbol{I}_{N} \otimes\left[\boldsymbol{\Theta}_{1} \boldsymbol{Q}+*\right]+\boldsymbol{I}_{N} \otimes \boldsymbol{S}_{1}+\boldsymbol{I}_{N} \otimes \boldsymbol{S}_{2} .
\end{gathered}
$$

In this case, the control gain matrices $\boldsymbol{K}_{1}$ and $\boldsymbol{K}_{2}$ of the controller of Equation (5) are:

$$
\boldsymbol{K}_{1}=\boldsymbol{Y}_{1} \boldsymbol{Q}^{-1} \text { and } \boldsymbol{K}_{2}=\boldsymbol{Y}_{2} \boldsymbol{Q}^{-1} \text {. }
$$

Proof. Assume that LMI of Equation (11) is satisfied for $n \times n$ symmetric matrices $\boldsymbol{Q}>0$, $\boldsymbol{S}_{1}>0, \boldsymbol{S}_{2}>0, \boldsymbol{S}_{3}>0$, and $m \times n$ matrices $\boldsymbol{Y}_{1}, \boldsymbol{Y}_{2}$. With application of Lemma 1, preand post-multiplying both sides of the matrix $\Xi$ by the block diagonal matrix $\mathcal{Q} \triangleq \operatorname{diag}\left\{\boldsymbol{I}_{N} \otimes \boldsymbol{Q}^{-1}, \boldsymbol{I}_{N} \otimes \boldsymbol{Q}^{-1}, \boldsymbol{I}_{N} \otimes \boldsymbol{Q}^{-1}, \boldsymbol{I}_{N} \otimes \boldsymbol{Q}^{-1}, \boldsymbol{I}_{N} \otimes \boldsymbol{Q}^{-1}\right\}$, respectively, and letting:

$$
Q=P^{-1}, Y_{1}=K_{1} Q, Y_{2}=K_{2} Q, Z=L Q, S_{1}=Q R_{1} Q, S_{2}=Q R_{2} Q, S_{3}=Q R_{3} Q,
$$

we obtain:

$$
\Psi=\mathcal{Q} \Xi \mathcal{Q}
$$

Since $\mathcal{Q}>0$, we can get from Equations (11) and (14) that:

$$
\Psi<0 .
$$

By virtue of the matrix theory and Equation (15), we can find an appropriate scalar $\rho \in \mathfrak{R}^{+}$such that:

$$
\Psi+\rho \boldsymbol{I} \leq 0 .
$$

It follows from Equation (16) that the inequality of Equation (9) can be rewritten as:

$$
\dot{V}(t) \leq-\rho\|\tilde{\boldsymbol{e}}(\cdot, t)\|_{2}^{2} \leq-\rho\|\boldsymbol{e}(\cdot, t)\|_{2}^{2} \text {, for } \boldsymbol{e}(\cdot, t) \neq 0 .
$$

By using the technique exploited in [36], the inequality of Equation (17) tells us that the closed-loop PDDE system of Equation (1) is exponentially stable if LMI of Equation (11) is fulfilled. From Definition 1, the closed-loop synchronization error system of Equation (6) is exponentially stable, i.e., the PDDE Equation (1) achieves projective exponential synchronization with the isolated node of Equation (2). Moreover, Equation (13) gives the expression of Equation (12). The proof is complete. 
On the basis of Lyapunov's direct method and integration by parts, Theorem 1 presents an LMI-based sufficient condition on the existence of a distributed state feedback controller of Equation (5) for projective exponential synchronization of the complex PDDE network of Equation (1). The explicit control gain matrices of the desired controller is given as Equation (12) when LMI of Equation (10) is feasible.

Remark 1. The distributed $P-s D$ controller is proposed for exponential stability of PDE systems in [37,38], while projective exponential synchronization of a complex PDDE network with multiple time delays and $N$ identical nodes was studied via the distributed P-sD control.

Remark 2. This paper considers the problem of projective exponential synchronization of a network considering multiple time delays. It is different from those in [34,35], in which the exponential synchronization problem of complex networks without time delays was discussed only.

Remark 3. The complex PDDE network in this paper contains time delays $\tau_{1}, \tau_{2}$ and $\tau_{3}$ without coupling time delay. A more complex model with coupling time delay will be further studied in future. Moreover, it should be pointed out that the method in this paper can also be extended to the network with spatially variable parameters. This will also be left for our future work.

\section{Simulation Study}

In this section, a chemical reactor network with four nodes is used to show the effectiveness of the proposed theoretical results. Every chemical reactor is a network node modeled by the linearized FitzHugh-Nagumo (FHN) equation with coupling terms, where FHN equations have been extensively employed for wavy behavior in chemistry. This reactor network can be given as Equation (1) with the following parameters:

$$
\begin{aligned}
& \boldsymbol{y}_{i}(x, t)=\left[y_{i 1}(x, t) \quad y_{i 2}(x, t)\right]^{T}, \\
& \Theta_{1}=\left[\begin{array}{ll}
1 & 0 \\
0 & 4
\end{array}\right], \Theta_{1 d}=\left[\begin{array}{cc}
-0.5 & 0 \\
0 & 0.5
\end{array}\right], \Theta_{2}=\left[\begin{array}{ll}
1 & 0 \\
0 & 1
\end{array}\right], \Theta_{2 d}=\left[\begin{array}{cc}
-1 & 0 \\
0 & 0.8
\end{array}\right], \quad A=\left[\begin{array}{cc}
2 & -0.2 \\
-1 & 3
\end{array}\right], \\
& \boldsymbol{A}_{d}=\left[\begin{array}{ll}
1 & 0 \\
0 & 1
\end{array}\right], \boldsymbol{B}=\left[\begin{array}{l}
1 \\
0
\end{array}\right], c=1, L=20, \tau_{1}=1, \tau_{2}=1.5, \tau_{3}=2, \tau=\max \left\{\tau_{1}, \tau_{2}, \tau_{3}\right\}=2 \text {, } \\
& \boldsymbol{G}=\left(g_{i j}\right)_{4 \times 4} \triangleq\left[\begin{array}{cccc}
-1.3592 & 0.3575 & 0.5155 & 0.4863 \\
-.2155 & 0.5622 & 0.3110 & -0.6576 \\
0.4121 & -0.9363 & 0.9704 & -0.4462 \\
-0.9077 & -0.8057 & 0.6469 & 1.0665
\end{array}\right] \text {. }
\end{aligned}
$$

The initial conditions are given as:

$$
\begin{gathered}
\phi(x, t)=\left[\begin{array}{c}
2 \cos (\pi x / L+2)+2 \sin ^{2}(\pi t) \\
2 \cos (2 \pi x / L)
\end{array}\right], \phi_{2}(x, t)=\left[\begin{array}{c}
3 \cos (\pi x / L) \\
2 \cos (3 \pi x / L+2)-3 \sin ^{3}(\pi t)
\end{array}\right], \\
\phi_{3}(x, t)=\left[\begin{array}{c}
5 \cos (2 \pi x / L)+4 \sin (-3 \pi t) \\
\cos ^{2}(\pi x / L)
\end{array}\right], \phi_{4}(x, t)=\left[\begin{array}{c}
8 \sin (0.5 \pi x / L+\pi) \\
2 \cos (-5 \pi x / L)+3 \sin ^{2}(2 \pi t)
\end{array}\right],
\end{gathered}
$$




$$
\boldsymbol{s}_{0}(x, t)=\left[\begin{array}{ll}
0.2 & 0.5
\end{array}\right]^{T}, t \in[-2,0]
$$

Figure 1 shows the open-loop profiles of evolution of $\boldsymbol{y}_{i}(x, t), i \in\{1,2,3,4\}$ for the complex PDDE network of Equation (1) with the parameters given in Equation (18) and the initial condition given in Equation (19). It is clear from Figure 1 that the nodes of the complex PDDE network are not synchronized.

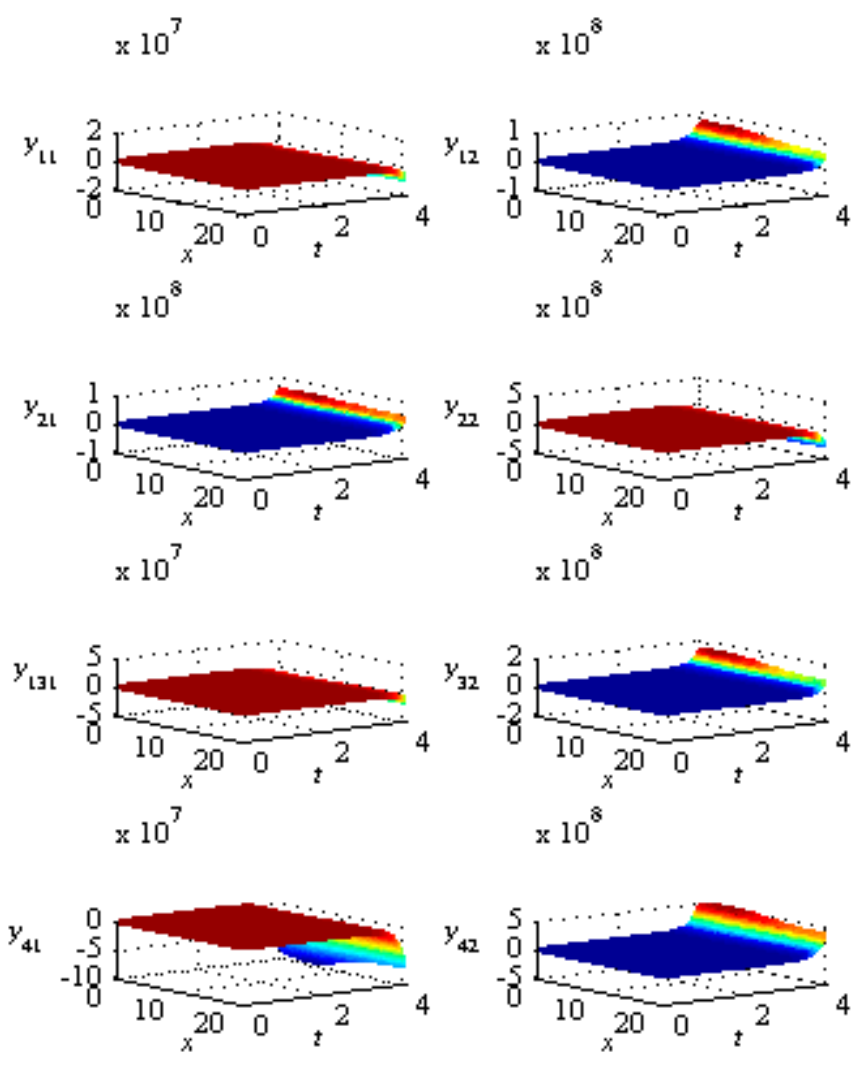

Figure 1. Open-loop profiles of evolution of $\boldsymbol{y}_{i}(x, t), i \in\{1,2,3,4\}$.

In this example, the controller of Equation (5) is employed, where $\boldsymbol{K}_{1} \triangleq\left[\begin{array}{ll}k_{11} & k_{12}\end{array}\right], \boldsymbol{K}_{2} \triangleq\left[\begin{array}{ll}k_{21} & k_{22}\end{array}\right]$ are $1 \times 2$ matrices to be determined. Divide the spatial domain [0,20] into space instances $\left\{x_{k}, k \in\{0,1,2, \cdots, 100\}, x_{0}=0, x_{100}=20\right\}$ of the same distance, where $x_{k}-x_{k-1}=20 / 100=0.2$. When setting $\alpha=-1$ and solving LMI of Equation (11), we get feasible solutions of $\boldsymbol{Q}>0, \boldsymbol{S}_{1}>0, \boldsymbol{S}_{2}>0$, $\boldsymbol{S}_{3}>0$, and $\boldsymbol{Y}_{1}, \boldsymbol{Y}_{2}$. By using of Equation (12), we further get the control gain matrices as follows:

$$
\boldsymbol{K}_{1} \triangleq\left[\begin{array}{ll}
-13.4733249 .6802
\end{array}\right] \text { and } \boldsymbol{K}_{2} \triangleq\left[\begin{array}{lll}
-0.9974 & -0.2529
\end{array}\right] \text {. }
$$

Applying the controller of Equation (5) with the obtained control parameters $\boldsymbol{K}_{1}$ and $\boldsymbol{K}_{2}$ Equation (20) to the complex PDDE network of Equation (1) with the parameters given in Equation (18) and the initial condition given in Equation (19), the closed-loop error profiles of evolution of $\boldsymbol{e}_{i}(x, t), i \in\{1,2,3,4\}$ are shown in Figure 2. Obviously, the proposed control law of Equation (5) can guide the complex PDDE network to projective exponentially synchronize with the isolated node $s(x, t)$. Moreover, Figure 3 shows the corresponding profiles of evolution of control inputs $u_{i}(x, t), i \in\{1,2,3,4\}$. 

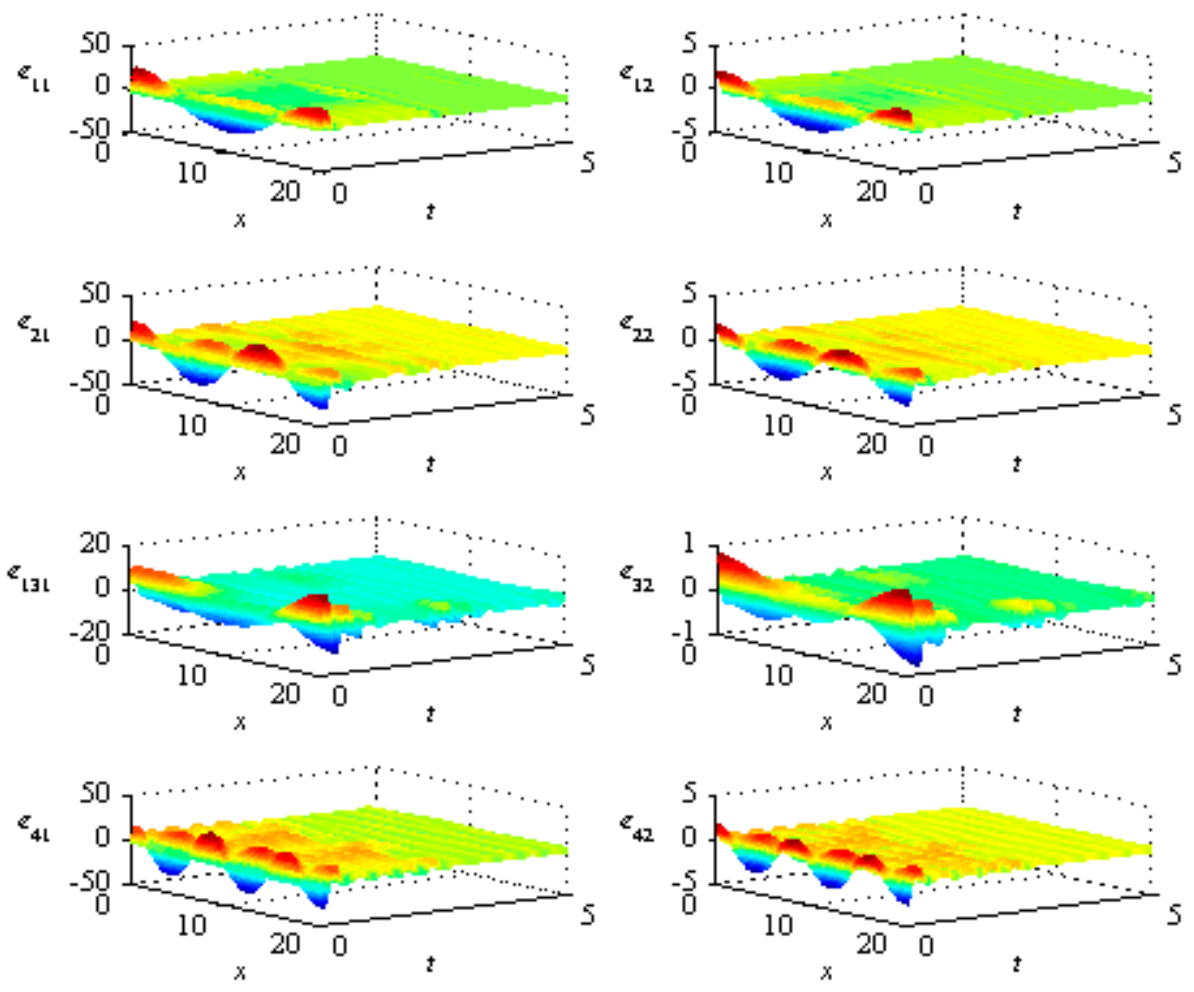

Figure 2. Close-loop error profiles of evolution of $\boldsymbol{e}_{i}(x, t), i \in\{1,2,3,4\}$.
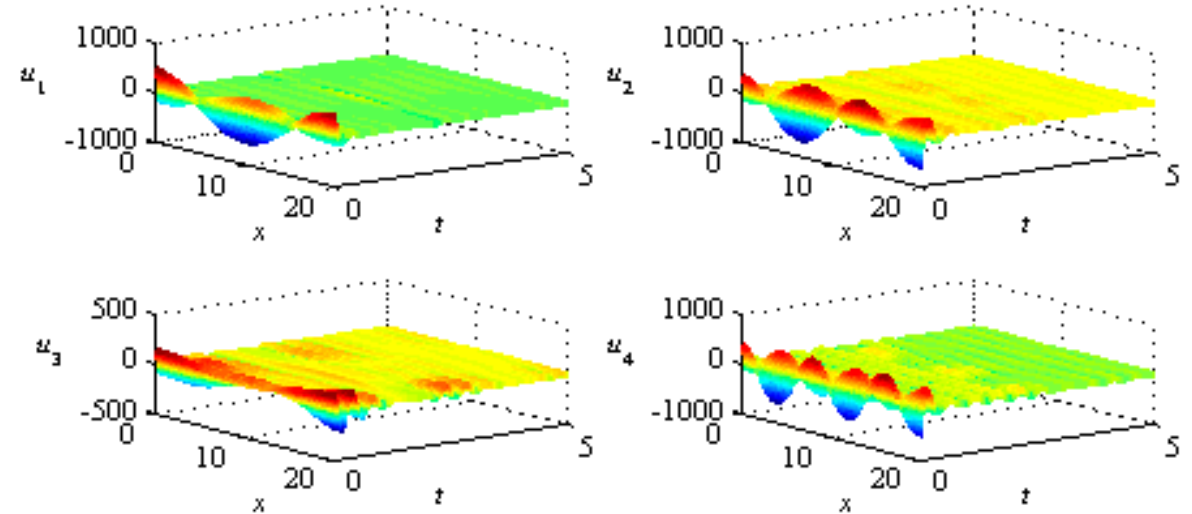

Figure 3. Profiles of evolution of control inputs $\boldsymbol{u}_{i}(x, t), i \in\{1,2,3,4\}$.

\section{Conclusions}

In this paper, we addressed the projective exponential synchronization problem of a class of complex PDDE networks. By using Lyapunov's direct method and the technique of integration by parts, a sufficient condition on the existence of distributed P-sD controller achieving the projective exponential synchronization was developed in terms of LMI. The control gain parameters are easily obtained via feasible solutions to the given LMI. Finally, the effectiveness of the proposed theoretical result is illustrated by a numerical example. It should be noted that the method in this paper can be extended to the network with spatially variable parameters as well as coupling time delay. This will be our future research topic. 


\section{Acknowledgments}

This work was supported in part by the National Natural Science Foundation of China under Grant 61273012, 61403179, 61304023 and 61503171, in part by the Natural Science Foundation of Shandong Province of China under Grant No. ZR2014AL009, ZR2014CP008 and ZR2015FL021, in part by the Science and Technology Development Plan Project of Shandong Province under Grant 2012YD01052, in part by 2015 Team Construction of Academic Degree of Linyi University under Grant 51715015, in part by 2015 Commercial Logistics Research Center Team of Linyi University under Grant 51715020 , and in part by the AMEP of Linyi University.

\section{Author Contributions}

Chengdong Yang contributed the design of the study and manuscript preparation; Jianlong Qiu contributed to the conception of the study; Tongxing Li performed the data analyses; Ancai Zhang and Xiangyong Chen helped perform the discussion and language description of this paper. All authors have read and approved the final manuscript.

\section{Conflicts of Interest}

The authors declare that they have no competing interests.

\section{References}

1. Cross, M.; Hohenberg, P. Pattern formation outside of equilibrium. Rev. Mod. Phys. 1993, 65, $851-1112$.

2. Cohen, M.; Grossberg, S. Absolute stability and global pattern formation and parallel memory storage by competitive neural networks. IEEE Trans. Syst. Man Cybern. 1983, 13, 815-826.

3. Marcus, C.; Westervelt, R. Stability of analog neural networks with delay. Phys. Rev. A 1989, 39, 347-359.

4. Cochocki, A.; Unbehauen, R. Neural Networks for Optimization and Signal Processing; Wiley: New York, NY, USA, 1993.

5. Bowong, S. Stability analysis for the synchronization of chaotic systems with different order: Application to secure communications. Phys. Lett. A 2004, 326, 102-113.

6. Cuomo, K.; Oppenheim, A.; Strogatz, S. Synchronization of Lorenz-based chaotic circuits with applications to communications. IEEE Trans. Circuits Syst. II 1993, 40, 626-633.

7. Yang, T.; Chua, L. Impulsive stabilization for control and synchronization of chaotic systems: Theory and application to secure communication. IEEE Trans. Circuits Syst. I 1997, 44, 976-988.

8. Boccaletti, S.; Kurths, J.; Osipov, G.; Valladares, D.L.; Zhou, C.S. The synchronization of chaotic systems. Phys. Rep. 2006, 366, 1-101.

9. Arenas, A.; Díaz-Guilera, A.; Kurths, J.; Moreno, Y.; Zhou, C. Synchronization in complex networks. Phys. Rep. 2008, 469, 93-153.

10. Eisencraft, M.; Fanganiello, R.; Monteiro, L. Chaotic synchronization in discrete-time systems connected by bandlimited channels. IEEE Commun. Lett. 2011, 15, 671-673. 
11. Nicosia, V.; Valencia, M.; Chavez, M.; Díaz-Guilera, A.; Latora, V. Remote synchronization reveals network symmetries and functional modules. Phys. Rev. Lett. 2013, 110, 174102.

12. Cao, J.; Ho, D.; Yang, Y. Projective synchronization of a class of delayed chaotic systems via impulsive control. Phys. Lett. A 2009, 373, 3128-3133.

13. Zhou, P.; Bai, R.; Zheng, J. Projective synchronization for a class of fractional-order chaotic systems with fractional-order in the $(1,2)$ interval. Entropy 2015, 17, 1123-1134.

14. Xin, B.; Wu, Z. Projective synchronization of chaotic discrete dynamical systems via linear state error feedback control. Entropy 2015, 17, 2677-2687.

15. Baldi, P.; Atiya, A. How delays affect neural dynamics and learning. IEEE Trans. Neural Netw. 1994, 5, 612-621.

16. Chapeau-Blondeau, F.; Chauvet, G. Stable, oscillatory, and chaotic regimes in the dynamics of small neural networks with delay. Neural Netw. 1992, 5, 735-743.

17. Bélair, J.; Campbell, S.; Driessche, P. Frustration, stability, and delay-induced oscillations in a neural network model. SIAM J. Appl. Math. 1996, 56, 245-255.

18. Cao, J.; Lu, J. Adaptive synchronization of neural networks with or without time-varying delay. Chaos 2006, 16, doi:10.1063/1.2178448.

19. Wang, Z.; Wang, Y.; Liu, Y. Global synchronization for discrete-time stochastic complex networks with randomly occurred nonlinearities and mixed time delays. IEEE Trans. Neural Netw. 2010, 21, $11-25$.

20. Zhou, J.; Xiang, L.; Liu, Z. Synchronization in complex delayed dynamical networks with impulsive effects. Physica A 2007, 384, 684-692.

21. Yu, W.; Cao, J. Synchronization control of stochastic delayed neural networks. Physica A 2007, $373,252-260$.

22. Yang, X.; Yang, Z. Synchronization of TS fuzzy complex dynamical networks with time-varying impulsive delays and stochastic effects. Fuzzy Sets Syst. 2014, 235, 25-43.

23. He, P.; Jing, C.; Fan, T.; Chen, C.-Z. Robust decentralized adaptive synchronization of general complex networks with coupling delayed and uncertainties. Complexity 2014, 19, 10-26.

24. Murray, J. Mathematical Biology; Springer: Berlin, Germany, 1989.

25. Grigoriev, R.; Cross, M.; Schuster, H. Pinning control of spatiotemporal chaos. Phys. Rev. Lett. 1997, 79, 2795-2798.

26. Parekh, N.; Parthasarathy, S.; Sinha, S. Global and local control of spatiotemporal chaos in coupled map lattices. Phys. Rev. Lett. 1998, 81, 1401-1404.

27. Wu, K.; Li, C.; Chen, B.; Yao, Y. Robust $H_{\infty}$ synchronization of coupled partial differential systems with spatial coupling delay. IEEE Trans. Circuits Syst. II 2013, 60, 451-455.

28. Wu, K.; Chen, B. Synchronization of partial differential systems via diffusion coupling. IEEE Trans. Circuits Syst. I 2012, 59, 2655-2668.

29. Wang, Z.; Zhang, H. Global asymptotic stability of reaction-diffusion Cohen-Grossberg neural networks with continuously distributed delays. IEEE Trans. Neural Netw. 2010, 21, 39-49.

30. Wang, J.; Wu, H.; Guo, L. Passivity and stability analysis of reaction-diffusion neural networks with Dirichlet boundary conditions. IEEE Trans. Neural Netw. 2011, 22, 2105-2116.

31. Codreanu, S. Synchronization of spatiotemporal nonlinear dynamical systems by an active control. Chaos Solitions Fractal 2003, 15, 507-510. 
32. Yuan, K.; Alofi, A.; Cao, J.; Al-Mazrooei, A.; Elaiw, A. Synchronization of the coupled distributed parameter system with time delay via proportional-spatial derivative control. Discret. Dyn. Nat. Soc. 2014, 2014, doi:10.1155/2014/418258.

33. Wang, J.; Yang, C.; Sun, C. Exponential synchronization for a class of networked linear parabolic PDE systems via boundary control. In Proceedings of the 2014 International Joint Conference on Neural Networks (IJCNN), Beijing, China, 6-11 July 2014; pp. 3981-3986.

34. Yang, C.; Qiu, J.; Yi, K.; Chen, X.; Zhang, A.; Chen, X.; Yang, L. Robust exponential synchronization for a class of master-slave distributed parameter systems with spatially variable coefficients and nonlinear perturbation. Math. Probl. Eng. 2015, doi:10.1155/2015/380903.

35. Yang, C.; Qiu, J.; He, H. Exponential synchronization for a class of complex spatio-temporal networks with space-varying coefficients. Neurocomputing 2015, 151, 401-407.

36. Mao, X.; Koroleva, N.; Rodkina, A. Robust stability of uncertain stochastic differential delay equations. Syst. Control Lett. 1998, 35, 325-336.

37. Wang, J.; Wu, H.; Li, H. Distributed proportional-spatial derivative control of nonlinear parabolic systems via fuzzy PDE modeling approach. IEEE Trans. Syst. Man Cybern. Part B 2012, 42 , 927-938.

38. Yang, C.; Qiu, J.; Wang, J. Robust $H_{\infty}$ control for a class of nonlinear distributed parameter systems via proportional-spatial derivative control approach. Abstr. Appl. Anal. 2014, 2014, doi:10.1155/2014/631071.

(C) 2015 by the authors; licensee MDPI, Basel, Switzerland. This article is an open access article distributed under the terms and conditions of the Creative Commons Attribution license (http://creativecommons.org/licenses/by/4.0/). 